\title{
Comparação entre a razão de viabilidade subendocárdica obtida por tonometria de aplanação e alterações de perfusão miocárdica pela cintilografia
}

\author{
Comparison between the subendocardial viability ratio obtained by applanation \\ tonometry and myocardial perfusion defects by scintigraphy
}

\author{
Francisco Eberth Marinho Marques ${ }^{1}$, Valéria Costa Hong ${ }^{2}$, \\ Maria Clementina Pinto Giorgi ${ }^{3}$, Luiz Aparecido Bortolotto ${ }^{4}$
}

\begin{abstract}
Marques FEM, Hong VC, Giorgi MCP, Bortolotto LA. Comparação entre a razão de viabilidade subendocárdica obtida por tonometria de aplanação e alterações de perfusão miocárdica pela cintilografia / Comparison between the subendocardial viability ratio obtained by applanation tonometry and myocardial perfusion defects by scintigraphy. Rev Med (São Paulo). 2014 jan.-mar.;93(1):14-21.
\end{abstract}

\begin{abstract}
RESUMO: Introdução: Estudos recentes correlacionam índices obtidos por análise do contorno da onda de pulso pela tonometria de aplanação com fatores de risco cardiovascular, rigidez arterial e aterosclerose. Destes índices, a razão de viabilidade subendocárdica (RVSE), obtida pela razão da área diastólica e a área sistólica da onda de pulso, é considerada uma estimativa de perfusão coronariana. No entanto, não há estudo da correlação da RVSE com provas de perfusão, como a cintilografia. Métodos: Foram avaliados 52 pacientes com indicação de cintilografia de perfusão miocárdica para avaliação de suspeita de doença coronariana, dos quais foram obtidas as medidas de tonometria de aplanação pelo aparelho SphygmoCor ${ }^{\circledR}$. Após a coleta dos dados, foi realizada a análise estatística para avaliar correlações entre os índices tonométricos e os achados cintilográficos. Resultados: O exame de cintilografia de perfusão miocárdica revelou alteração em 23 indivíduos (44,2\%), sendo mais frequente em homens e naqueles com mais de 4 fatores de risco associados. A RVSE foi estatisticamente semelhante nos pacientes com $(162,4 \pm 22,7 \%)$ ou sem alteração $(164,7 \pm 32,2 \%)$ na cintilografia de perfusão miocárdica, mesmo considerando apenas as alterações isquêmicas transitórias. Os demais índices obtidos pela tonometria também não mostraram diferença entre os grupos com
\end{abstract}

\begin{abstract}
ou sem alteração na cintilografia. Conclusões: Em pacientes com suspeita de doença arterial coronariana observou-se mais alterações de perfusão na cintilografia miocárdica em homens e em pacientes com múltiplos fatores de risco. A RVSE assim como os demais índices obtidos pela análise da onda de pulso não se correlacionaram com alterações de perfusão miocárdica da cintilografia.
\end{abstract}

DESCRITORES: Análise de onda de pulso; Imagem de perfusão do miocárdio; Manometria; Rigidez vascular; Cintilografia.

\begin{abstract}
Introduction: Recent research correlates indices acquired by pulse wave analysis with cardiovascular risk factors, arterial stiffness and atherosclerosis. One of them, the subendocardial viability ratio (SEVR), obtained from the ratio between the diastolic and systolic area of the pulse wave, has been considered as an estimative of coronary perfusion. However, there is no record of comparison between SEVR and other tools of myocardial perfusion as the scintigraphy. Methods: We evaluated 52 patients referred for myocardial perfusion imaging to assess the suspicion of coronary artery disease, followed by the applanation tonometry which was performed in these attendees by
\end{abstract}

Prêmio Oswaldo Cruz - Área Clínica - COMU 2013 - XXXII Congresso Médico Universitário da FMUSP.

1. Acadêmico do curso de Medicina da Faculdade de Medicina da Universidade de São Paulo,aluno de iniciação científica da Unidade de Clínica de Hipertensão do InCor-HCFMUSP. E-mail: fcoeberth@hotmail.com.

2. Biologista da Unidade de Hipertensão do InCor-HCFMUSP. E-mail: hong.valeria@gmail.com.

3. Médica assistente da Divisão de Medicina Nuclear do Instituto do Coração da Faculdade de Medicina da Universidade de São Paulo. E-mail: mcp.giorgi@incor.usp.br.

4. Professor livre-docente e Professor colaborador médico no Departamento de Cardiopneumologia, Disciplina de Cardiologia da Faculdade de Medicina da Universidade de São Paulo; Diretor da Unidade de Hipertensão do InCor - HCFMUSP; Programa de Tutoria da FMUSP. E-mail: hipluiz@incor.usp.br.

Endereço para correspondência: Francisco E. M. Marques. InCor-HCFMUSP, Unidade de Hipertensão. Av. Dr. Enéas de Carvalho Aguiar, 44 - São Paulo, SP. CEP: 05403-900. 
SphygmoCor ${ }^{\circledR}$ device. After collecting the data, it was performed a statistical analysis to evaluate whether there was a correlation between tonometrical indices and scintigraphic findings. Results: Myocardial perfusion imaging revealed defects in 23 individuals studied (44.2\%), being more frequent in men and those with more than four associated risk factors. The SEVR was statistically similar between the patients with $(162.4 \pm 22.7 \%)$ or without $(164.7 \pm 32.2 \%)$ a defect in myocardial perfusion scintigraphy. Other indices measured by tonometry have not also presented correlation between the groups with or without defects in

\section{INTRODUÇÃO}

$\mathbf{R}$ ecentes estudos epidemiológicos em diferentes populações têm demonstrado uma associação mais importante entre a pressão de pulso, diferença entre a pressão sistólica e diastólica, e complicações cardiovasculares, sobretudo infarto do miocárdio e acidente vascular cerebral, do que as medidas isoladas da pressão sistólica ou diastólica ${ }^{1-4}$. Enquanto a pressão arterial média é quase constante ao longo da árvore arterial, a pressão de pulso aumenta significativamente das artérias mais centrais para as artérias periféricas, indicando que, in vivo, cada artéria deve ser caracterizada de acordo com sua própria curva de pressão $0^{5}$ Novos métodos não invasivos tem permitido uma avaliação mais precisa da pressão de pulso e da onda de pulso em diferentes territórios arteriais $^{6,7}$ e recentes estudos utilizando esta metodologia tem demonstrado correlações significativas entre as pressões de pulso locais e complicações vasculares em diferentes situações clínicas ${ }^{8,9,10}$.

A metodologia utilizada nestes estudos é baseada em tonometria de aplanação, já validada por diversos autores para medidas intra-arteriais ${ }^{6,711}$. Além da medida da pressão de pulso, cálculos matemáticos obtidos a partir da análise da onda de pulso têm permitido a obtenção de índices de rigidez arterial, tais como o índice de incremento ou "augmentation index”, a razão de viabilidade subendocárdica (RVSE), também conhecida como índice de Buckberg e o índice de duração de ejeção.

Alguns estudos, avaliando o índice de incremento e a pressão central obtidos pela tonometria, mostraram correlação dos mesmos com maior risco cardiovascular e com a presença de aterosclerose ${ }^{12,13,14}$. No entanto, poucos estudos têm avaliado a real importância clínica dos demais índices obtidos pela tonometria, tais como os relacionados à viabilidade subendocárdica. Em um destes estudos, avaliouse a correlação da RVSE com fatores de risco para a doença isquêmica do coração ${ }^{15}$. Os autores demonstraram que alterações da RVSE, obtidas pela tonometria de aplanação, eram influenciadas por vários fatores de risco para doença coronariana, tais como hipertensão, diabetes e dislipidemia. scintigraphy. Conclusion: In patients with suspicion of coronary artery disease, it was observed more changes in perfusion on myocardial scintigraphy in men and in patients with multiple risk factors. SEVR as well as others arterial stiffness indices obtained by pulse wave analysis from applanation tonometry have not correlated with myocardial perfusion imaging defect.

KEYWORDS: Pulse wave analysis; Myocardial perfusion imaging; Manometry; Vascular stiffness; Radionuclide imaging.

No entanto, não se correlacionou estas alterações com outras provas de perfusão miocárdica. Nesse contexto, a cintilografia de perfusão miocárdica é considerada um dos métodos não invasivos mais precisos para determinar alterações de perfusão e viabilidade miocárdica de pacientes com aterosclerose coronariana, ou mesmo em outras patologias que interferem na circulação cardíaca ${ }^{16}$.

Até o momento não há estudos na literatura que tenham comparado os diferentes índices obtidos pela tonometria de aplanação com alterações de perfusão miocárdica observadas na cintilografia induzida por estresse. Desse modo, o objetivo desse estudo é avaliar se a RVSE se correlaciona com alterações cintilográficas de perfusão miocárdica em pacientes com suspeita clínica de doença arterial coronariana.

\section{MATERIAL E MÉTODOS}

População do estudo: Foram estudados 52 pacientes (idade média 63,8 111,0 anos, sendo 29 (55,8\%) homens) com indicação de cintilografia de perfusão miocárdica para avaliar a suspeita de doença arterial coronariana. Critérios de exclusão incluíram: valvopatia, arritmia cardíaca (fibrilação atrial), bloqueio de ramo intraventricular ou qualquer outra condição clínica que resultasse em índice de qualidade da curva obtida pela tonometria de aplanação abaixo de $80 \%$. Um questionário sobre fatores de risco cardiovasculares (dentre os quais: gênero e idade, hipertensão, diabetes, dislipidemia, tabagismo, história familiar de doença cardiovascular, sedentarismo e obesidade) foi respondido por todos os participantes. O protocolo do estudo que incluía o questionário de fatores de risco cardiovasculares, a obtenção da curva de tonometria de aplanação e a realização da cintilografia de perfusão miocárdica, respeitou a Declaração de Helsinki e foi aprovado pelo Comitê de Ética da Instituição onde foi realizada a pesquisa. Todos participantes assinaram termo de consentimento livre e esclarecido após descrição detalhada sobre o estudo.

Medida do peso, altura e pressão arterial: o peso e a altura de cada paciente foram verificados com balança digital padronizada com estadiômetro acoplado. A obtenção da 
medida de pressão arterial braquial (sistólica e diastólica), aferida por aparelho automático validado (Omron 705CP; Omron ${ }^{\circledR}$, Kyoto, Japão), foi realizada a partir da média aritmética de três medidas com intervalos de 2 minutos cada, após cinco minutos de repouso na posição sentada, de acordo com as recomendações da VI Diretrizes Brasileiras de Hipertensão ${ }^{17}$. As medidas descritas acima foram realizadas antes da determinação da curva de tonometria de aplanação de cada paciente.

Tonometria de aplanação: Esta avaliação foi realizada a partir do aparelho SphygmoCor ${ }^{\circledR}$ (AtCor Medical ${ }^{\circledR}$, Sidney, Austrália), que utiliza como base a tecnologia da tonometria de aplanação, que permite estudar as diferenças de pressão sistólica e diastólica intra-arteriais a partir da aplicação de um dispositivo semelhante a uma caneta, com um micromanômetro na ponta, sobre o trajeto da artéria interessada, que neste caso foi a artéria radial. Todos pacientes submeteram-se ao registro das medidas da tonometria de aplanação antes da realização da cintilografia de perfusão miocárdica. Para a realização do procedimento, os pacientes mantiveram-se sentados, com o braço onde seria realizada a medida na posição horizontal e apoiado em um coxim sobre uma mesa. A pressão exercida pelo operador do dispositivo para obter a curva tonométrica foi aquela suficiente para não ocluir a artéria radial. Os valores de pressão arterial sistólica e diastólica, peso e altura utilizados para calibragem do aparelho foram obtidos de acordo com tópico específico descrito anteriormente. Foram obtidas duas curvas de tonometria de cada participante e os valores utilizados no estudo foram a média aritmética dos parâmetros encontrados. Os registros obtidos foram transferidos instantaneamente para o software do aparelho (previamente instalado em um com- putador acoplado ao dispositivo que realiza as medidas), o qual deriva a onda de pressão da aorta ascendente (pressão central) a partir da onda de pressão da artéria radial, além de determinar propriedades específicas do ventrículo esquerdo (RVSE e duração de ejeção), separar a onda aórtica em períodos sistólico e diastólico e determinar índices de interação ventricular-vascular (pressão de incremento e índice de incremento, por exemplo) a partir de fórmulas matemáticas também derivadas da onda de pressão da artéria radial. O registro das curvas foi realizado por operador único treinado e o índice de qualidade (que indica reprodutibilidade da onda registrada) para que a curva fosse considerada satisfatória para o estudo foi de $80 \%$, de acordo com as especificações do aparelho.

Cálculo da RVSE e demais índices tonométricos: todos os índices obtidos pela tonometria de aplanação (RVSE, duração de ejeção, pressão de incremento ou "augmentation pressure" (AP), índice de incremento ou "augmentation index" (AIx), pressão de pulso central (PPc), pressão sistólica e diastólica de aorta ascendente foram derivados de fórmulas matemáticas obtidas a partir da curva de onda de pressão da artéria radial. A RVSE, também conhecida como Índice de Buckberg, de acordo com a sua definição clássica, é uma razão gerada a partir da integral pressão-tempo obtida a partir de pressões na aorta e ventrículo esquerdo, porém no presente estudo, de forma simples, pode ser definida como: RVSE = área aórtica diastólica/ área aórtica sistólica, e foi automaticamente calculada pelo programa do aparelho SphygmoCor $®$ (Figura 1). Aárea diastólica representada no numerador reflete a perfusão miocárdica (oferta de oxigênio), enquanto a área sistólica indicada no denominador caracteriza a contração miocárdica (demanda por oxigênio).

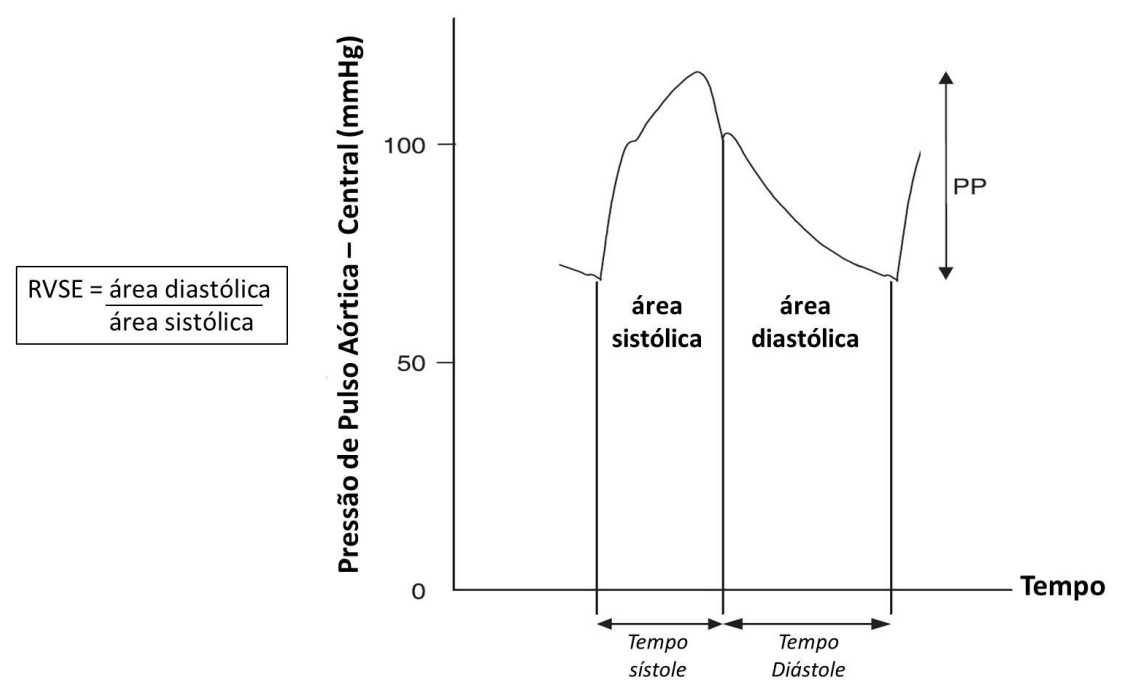

Figura 1 - Representação gráfica da Razão de Viabilidade Subendocárdica - RVSE (adaptado de Clin Exp Pharmacol Physiol. Subendocardial viability ratio estimated by arterial tonometry: a critical evaluation in elderly hypertensive patients with increased aortic stiffness. p. 910) 
O AIx, medida que reflete a rigidez arterial, é expresso como uma porcentagem da PPc (Aix $=\mathrm{AP} / \mathrm{PPc}$ x $100 \%$ ) decorrente da reflexão da onda de pulso que percorre o território arterial, de modo que gera um incremento à onda de pulso inicial (Figura 2). Por variar com a frequência cardíaca (FC), o AIx é ajustado pelo próprio aparelho para a frequência de 75 batimentos por minuto, independente da FC apresentada pelo paciente, gerando o AIx-75. A AP, expressa em $\mathrm{mmHg}$, corresponde ao valor absoluto de pressão que é acrescida ao valor inicial da onda de pressão após a reflexão desta. A PPc, também indicada em mmHg, é calculada a partir da diferença entre a pressão sistólica aórtica e a pressão diastólica aórtica.
A duração de ejeção, como o nome indica, reflete a porcentagem do tempo do ciclo cardíaco que corresponde à ejeção ventricular, de modo que representa indiretamente a função cardíaca sistólica e diastólica. Os valores de referência para pressão sistólica aórtica, pressão diastólica aórtica, PPc, AP e AIx variavam de acordo com a idade e sexo do paciente, enquanto que a RVSE e a duração de ejeção eram influenciadas pela frequência cardíaca. Para todos esses índices, o programa apresentava uma curva com os intervalos de uma população previamente estudada (valores 5\% inferiores, 90\% intermediários e 5\% superiores, de acordo com sexo e idade ou a FC, dependendo do índice estudado).

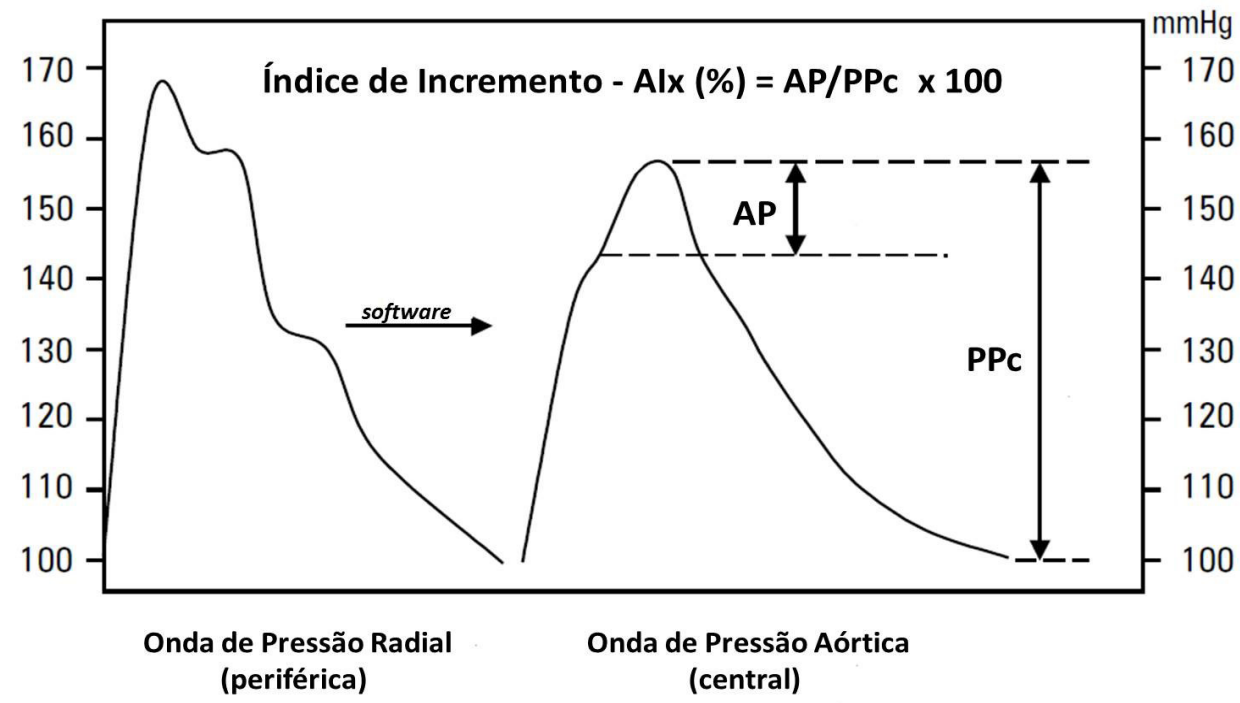

Figura 2 - Representação gráfica do Índice de Incremento - AIx (adaptado de Med Sci Monit. Measurement and application of arterial stiffness in clinical research: focus on new methodologies and diabetes mellitus. p. RA86)

Cintilografia de Perfusão Miocárdica: a cintilografia de perfusão miocárdica foi realizada em repouso e após estímulo farmacológico com adenosina. As imagens foram adquiridas em uma gama câmara equipada com dois detectores e colimadores de baixa energia e alta resolução dedicada a exames cardíacos (Phillips - CardioMD modelo I, Hoersholm, Dinamarca) com 64 projeções em uma órbita de $180^{\circ}$ aproximadamente uma hora após a administração do radiofármaco. As imagens foram sincronizadas ao eletrocardiograma (ECG) para análise da motilidade e cálculo da fração de ejeção do ventrículo esquerdo (FEVE) na fase de estresse. Inicialmente foram realizadas as imagens de repouso para a qual os pacientes receberam cerca de $370 \mathrm{MBq}$ de 2-metoxi 2-isobutil isonitrila marcada com tecnécio-99m (MIBI). Após as imagens de repouso os pacientes foram submetidos a estresse e receberam mais uma dose de MIBI (cerca de $1 \mathrm{GBq}$ ) seguida de nova série de imagens. Estas foram reconstruídas pelo método iterativo de medicina nuclear OSEM (Ordered Subset Expectation Maximisation - 12 iterações) e apresentadas nos eixos curto, longo horizontal e longo vertical do ventrículo esquerdo. Foi utilizada análise visual sendo atribuído um escore de defeito de captação do radiofármaco (0-normal; 1-hipocaptação discreta; 2- hipocaptação moderada; 3-hipocaptação acentuada; 4-ausência de captação) para cada segmento miocárdico em cada fase do exame (repouso e estresse) de acordo com o preconizado pela American Heart Association $^{18}$. As imagens reconstruídas foram processadas utilizando o programa AutoQuant (versão 5.0, Warrendale, PA, Estados Unidos) para análise da função do ventrículo esquerdo. A classificação dos defeitos de perfusão em persistente (sugestivo de fibrose ou necrose), transitório (sugestivo de isquemia) ou misto pela cintilografia de perfusão miocárdica foi baseada no escore de defeito de captação do radiofármaco. Desse modo, o defeito persistente é aquele no qual o escore de repouso (summed rest score - SRS) é maior que zero e a diferença (summed diference score - SDS) entre o escore no estresse (summed stress score - SSS) e o escore no repouso $(S R S)$ seja igual a zero; o defeito transitório é aquele em que a diferença $(S D S)$ entre o escore no estresse ( $S S S$ ) e o escore no repouso $(S R S)$ é maior que zero; e, por fim, o defeito misto é aquele que 
Marques FEM et al. Comparação entre a razão de viabilidade subendocárdica obtida por tonometria.

apresenta $S D S$ e $S R S$ maiores que zero (Tabela 1).

Tabela 1 - Valores de SDS, SSS e SRS de acordo com o defeito de perfusão (SDS=SSS-SRS)

\begin{tabular}{lccc}
\hline Defeito de Perfusão & SSS & SRS & $\begin{array}{c}\boldsymbol{S D S}=\boldsymbol{S S S} \\
-\boldsymbol{S R S}\end{array}$ \\
\hline Defeito Persistente & $>0$ & $>0(=\mathrm{SSS})$ & 0 \\
Defeito Transitório & $>0$ & 0 & $>0(=\mathrm{SSS})$ \\
Defeito Misto & $>0$ & $>0(<\mathrm{SSS})$ & $>0$ \\
\hline
\end{tabular}

Legenda: SDS (summed difference score), SSS (summed stress score), SRS (summed rest score).

Análise Estatística: $a$ análise estatística foi realizada a partir do programa JMP, versão 6.0 para Windows (JMP, SAS Institute Inc., Cary, NC, Estados Unidos). As variáveis contínuas foram expressas apresentando sua média e desvio-padrão, enquanto as variáveis categóricas foram expressas em porcentagem. Foi utilizado Teste t de Student (paramétrico) para variáveis contínuas entre dois grupos. Para as variáveis categóricas, utilizou-se o teste de $\chi^{2}$. $\mathrm{P}<0,05$ foi considerado estatisticamente significante.

\section{RESULTADOS}

Inicialmente foram incluídos 62 pacientes com indicação de cintilografia de perfusão miocárdica, porém 10 deles $(16,1 \%)$ foram excluídos por apresentarem condições que impediam a obtenção de curva de tonometria de aplanação com a qualidade mínima necessária de $80 \%$. Dentre os excluídos, oito participantes apresentavam arritmia, um deles valvopatia, e o último excluído apresentava pulso filiforme, de modo que o operador do aparelho não conseguiu registro de curva satisfatória.

Dentre a população final de 52 pacientes estudados, a cintilografia de perfusão miocárdica revelou alteração em 23 indivíduos (44,2\%), sendo 11 deles $(47,8 \%)$ com defeito persistente, $7(30,4 \%)$ com defeito transitório e $5(21,7 \%)$ com defeito misto (Tabela 2).

Tabela 2 - Pacientes com ou sem defeito de perfusão em cintilografia miocárdica e distribuição daqueles com defeito de acordo com o tipo

\begin{tabular}{ll}
\hline Cintilografia de Perfusão Miocárdica & n (\%) \\
\hline Cintilografia Normal & $\mathbf{2 9}(\mathbf{5 5 , 8 )}$ \\
Defeito de Perfusão & $\mathbf{2 3 ( 4 4 , 2 )}$ \\
Defeito Persistente & $11(21,2)$ \\
Defeito Transitório & $7(13,4)$ \\
Defeito Misto & $5(9,6)$ \\
\hline
\end{tabular}

Legenda: Cintilografia normal - indica que não há nenhum defeito persistente e/ou transitório na cintilografia de perfusão miocárdica; Defeito de perfusão - indica que há algum defeito persistente e/ou transitório na cintilografia de perfusão miocárdica.
Análise comparativa de características clínicas (apresentadas com média e desvio-padrão), como idade, gênero, peso, altura, IMC e medidas de pressão arterial periférica, não foram diferentes entre o grupo com cintilografia normal ou aqueles com defeito de perfusão (Tabela 3), exceto pelo gênero masculino, percentualmente mais prevalente no grupo que apresentava defeito de perfusão (73,9\% vs. $41,4 \%, p<0,05)$.

Tabela 3 - Características clínicas encontradas nos pacientes com cintilografia de perfusão miocárdica com ou sem defeito de perfusão

\begin{tabular}{lccc}
\hline Variáveis & $\begin{array}{c}\text { Cintilografia } \\
\text { Normal } \\
(\mathbf{n}=\mathbf{2 9})\end{array}$ & $\begin{array}{c}\text { Defeito de } \\
\text { Perfusão } \\
(\mathbf{n = 2 3})\end{array}$ & $\mathbf{P}$ \\
\hline Idade (anos) & $63,4 \pm 12,9$ & $64,3 \pm 8,1$ & 0,77 \\
\hline $\begin{array}{l}\text { Gênero } \\
\text { masculino (\%) }\end{array}$ & 41,4 & 73,9 & 0,03 \\
\hline Peso (Kg) & $71,1 \pm 12,5$ & $74,5 \pm 18,5$ & 0,45 \\
\hline Altura (m) & $1,60 \pm 9,2$ & $1,63 \pm 10,3$ & 0,25 \\
\hline $\boldsymbol{I M C}\left(\mathbf{K g} / \boldsymbol{m}^{2}\right)$ & $27,8 \pm 5,1$ & $28,3 \pm 8,0$ & 0,82 \\
\hline $\boldsymbol{P A S}(\mathbf{m m H g})$ & $136,7 \pm 24,3$ & $135,3 \pm 13,0$ & 0,80 \\
\hline $\boldsymbol{P A D}(\mathbf{m m H g})$ & $79,9 \pm 12,0$ & $78,3 \pm 9,7$ & 0,60 \\
\hline $\boldsymbol{P P}(\mathbf{m m H g})$ & $56,8 \pm 24,0$ & $57,0 \pm 15,0$ & 0,70 \\
\hline
\end{tabular}

Legenda: Cintilografia normal - indica que não há nenhum defeito fixo e/ou transitório na cintilografia de perfusão miocárdica; Defeito de perfusão - indica que há algum defeito fixo e/ou transitório na cintilografia de perfusão miocárdica; IMC - índice de massa corpórea; PAS - pressão arterial braquial sistólica; PAD - pressão arterial braquial diastólica; PP - pressão de pulso braquial (diferença entre a pressão arterial braquial sistólica e diastólica).

A presença de fatores de risco cardiovascular também foi pesquisada entre o grupo com ou sem defeito de perfusão, e nesta análise o fator de risco tabagismo foi o único a mostrar tendência com a presença de alteração no exame de cintilografia (41,4 vs. $69,6 \%, p=0,05)$; os demais fatores de risco (gênero e idade, hipertensão, diabetes, dislipidemia, história familiar de doença cardiovascular, sedentarismo e obesidade) foram semelhantes entre os grupos estudados (Tabela 4).

A prevalência de fatores de risco cardiovascular nos pacientes com defeito de perfusão também foi estudada a partir da associação destes, de modo que pacientes com número de fatores de risco igual ou superior a 5 apresentaram percentualmente mais alteração no exame cintilográfico do que aqueles com 4 ou menos fatores $(21,7$ vs. $62,1 \%$, $\mathrm{p}=0,005)$ (Tabela 5).

As variáveis de tonometria de aplanação obtidas pelo aparelho SphygmoCor ${ }^{\circledR}$ entre os 52 pacientes pesquisados, com seus valores médios e desvios-padrão, são apresentadas a seguir (Tabela 6). 
Análise comparativa dos índices obtidos pela tonometria de aplanação também foi realizada considerando os grupos com cintilografia normal e aqueles com defeito de perfusão. A RVSE, principal índice avaliado no estudo, foi semelhante nos dois grupos de indivíduos $(164,7 \pm 32$ vs. $162,3 \pm 22 \%$; $=0,77)$. Os demais índices obtidos pela tonometria, como a AP, PPc, AIx, AIx-75, duração de ejeção, pressão sistólica aórtica e pressão diastólica aórtica também não foram diferentes entre os grupos com ou sem alteração na cintilografia (Tabela 7).

Tabela 4 - Fatores de risco cardiovascular clássicos e a relação destes com o resultado da cintilografia de perfusão miocárdica (normal ou com defeito de perfusão)

\begin{tabular}{lccc}
\hline $\begin{array}{l}\text { Fatores de Risco } \\
\text { Cardiovascular }\end{array}$ & $\begin{array}{c}\text { Cintilografia } \\
\text { Normal } \\
(\mathbf{n = 2 9 )}\end{array}$ & $\begin{array}{c}\text { Defeito de } \\
\text { Perfusão } \\
(\mathbf{n = 2 3 )}\end{array}$ & $\mathbf{P}$ \\
\hline Gênero e Idade (\%) & 86,2 & 100,0 & 0,12 \\
\hline HAS (\%) & 75,9 & 87,0 & 0,48 \\
\hline DM (\%) & 31,0 & 30,4 & 1,00 \\
\hline Dislipidemia (\%) & 65,6 & 78,3 & 0,22 \\
\hline Tabagismo (\%) & 41,4 & 69,6 & $\mathbf{0 , 0 5}$ \\
\hline $\begin{array}{l}\text { História Familiar } \\
\text { (\%) }\end{array}$ & 31,0 & 43,4 & 0,38 \\
\hline Obesidade (\%) & 20,1 & 34,8 & 0,35 \\
\hline Sedentarismo (\%) & 62,1 & 69,6 & 0,77 \\
\hline
\end{tabular}

Legenda: Fatores de risco cardiovascular (respostas a questionário): 1) Gênero e idade- se homem com idade maior que 45 anos ou mulher com idade maior que 55 anos; 2) HAS- hipertensão arterial sistêmica, se paciente com diagnóstico de HAS ou uso de anti-hipertensivo; 3) DM- diabetes melitus, se paciente com diagnóstico de DM, uso de hipoglicemiante oral ou insulina; 4) Dislipidemia- se paciente referisse colesterol/triglicérides elevado ou uso de medicamento para controle de dislipidemia; 5) Tabagismo- se referisse ser fumante ou ter parado de fumar; 6) História Familiar- se possuísse familiar de primeiro grau (homem com idade menor a 55 anos ou mulher com idade menor a 65 anos) com diagnóstico de alguma doença cardiovascular; 7) Obesidade- se IMC $\geq$ $30 \mathrm{~kg} / \mathrm{m}^{2} ; 8$ ) Sedentarismo - se atividade física abaixo de 90 minutos/ semana e 3 dias/semana.

Tabela 5 - Associação entre número de fatores de risco cardiovascular e presença de defeito de perfusão em cintilografia de perfusão miocárdica $(\mathrm{p}=0,005)$

\begin{tabular}{l}
\hline \\
$\begin{array}{l}\text { Número de fatores de } \\
\text { risco cardiovascular }\end{array}$
\end{tabular}

Tabela 6 - Variáveis obtidas a partir da tonometria de aplanação (SphygmoCor ${ }^{\circledR}$ ) com seus valores médios e desvios-padrão

\begin{tabular}{lc}
\hline Variáveis da Tonometria de Aplanação & $\begin{array}{c}\text { Média e DP } \\
(\mathbf{n}=\mathbf{5 2})\end{array}$ \\
\hline RVSE (\%) & $163,4 \pm 27,1$ \\
\hline DE (\%) & $33,8 \pm 3,6$ \\
\hline AP (mmHg) & $14,9 \pm 8,9$ \\
\hline PPc (mmHg) & $46,3 \pm 18,9$ \\
\hline AIx (\%) & $30,3 \pm 10,2$ \\
\hline AIx-75 (\%) & $26,5 \pm 8,6$ \\
\hline PSA (mmHg) & $126,2 \pm 18,2$ \\
\hline PDA (mmHg) & $79,6 \pm 11,3$ \\
\hline
\end{tabular}

Legenda: RVSE - razão de viabilidade subendocárdica; DE - duração de ejeção; AP - pressão de incremento; PPc - pressão de pulso aórtica; AIx - índice de incremento; AIx-75 - índice de incremento ajustado para 75 batimentos por minuto; PSA - pressão sistólica aórtica; PDA - pressão diastólica aórtica.

Tabela 7 - Correlação entre variáveis medidas pela tonometria de aplanação e resultado obtido pela cintilografia de perfusão miocárdica

\begin{tabular}{lccc}
\hline $\begin{array}{l}\text { Tonometria de } \\
\text { Aplanação }\end{array}$ & $\begin{array}{l}\text { Cintilografia } \\
\text { Normal (n=29) }\end{array}$ & $\begin{array}{c}\text { Defeito de } \\
\text { Perfusão } \\
(\mathbf{n = 2 3 )}\end{array}$ & P \\
\hline RVSE (\%) & $162,4 \pm 22,7$ & $164,7 \pm 32,2$ & 0,77 \\
\hline DE (\%) & $33,9 \pm 3,4$ & $33,7 \pm 3,9$ & 0,81 \\
\hline AP (mmHg) & $15,0 \pm 10,7$ & $14,9 \pm 5,9$ & 0,97 \\
\hline PPc (mmHg) & $46,9 \pm 17,0$ & $46,3 \pm 19,0$ & 0,70 \\
AIx (\%) & $29,8 \pm 11,7$ & $31,0 \pm 8,0$ & 0,67 \\
\hline AIx-75 (\%) & $26,1 \pm 9,4$ & $26,9 \pm 7,6$ & 0,76 \\
PSA (mmHg) & $127,0 \pm 22,0$ & $125,2 \pm 12,4$ & 0,70 \\
\hline PDA (mmHg) & $80,1 \pm 12,5$ & $78,9 \pm 9,9$ & 0,69 \\
\hline
\end{tabular}

Legenda: Cintilografia normal - indica que não há nenhum defeito persistente e/ou transitório na cintilografia de perfusão miocárdica; Defeito de perfusão - indica que há algum defeito persistente e/ou transitório na cintilografia de perfusão miocárdica; RVSE - razão de viabilidade subendocárdica; DE - duração de ejeção; AP aórtica - pressão de incremento; PPc - pressão de pulso aórtica; AIx - índice de incremento; AIx-75 - índice de incremento ajustado para 75 batimentos por minuto; PSA - pressão sistólica aórtica; PDA aórtica - pressão diastólica aórtica.

\section{DISCUSSÃO}

Nosso estudo avaliou, pela primeira vez na literatura, se os índices de análise da onda de pulso arterial obtidos pela tonometria de aplanação, em destaque para a RVSE, se correlacionavam com alterações de perfusão miocárdica, obtidas pela cintilografia com estresse farmacológico em pacientes com suspeita de doença arterial coronariana. Não encontramos nenhuma diferença entre os grupos que 
apresentavam ou não alteração de perfusão, mesmo considerando apenas as alterações transitórias.

Desde que o primeiro trabalho relacionando a razão entre a área diastólica e a área sistólica do contorno da onda de pulso foi publicado por Buckberg ${ }^{19}$, mostrando a relação deste índice com o balanço entre oferta e demanda de oxigênio subendocárdica, diferentes estudos que investigam a presença de aterosclerose subclínica a partir de marcadores derivados da onda de pulso, em pacientes de risco cardiovascular intermediário, foram publicados ${ }^{12,13,14}$. Apesar da RVSE, o marcador descrito por Buckberg, não ter sido muito valorizado nesses estudos, trabalhos mais recentes apontam correlação da RVSE com alterações do fluxo de reserva avaliado durante cinecoronariografia em pacientes sem doença coronariana ${ }^{20}$, com isquemia induzível em indivíduos submetidos a ecocardiograma em estresse por dobutamina ${ }^{21}$ e com maior demanda metabólica miocárdica em pacientes com choque séptico que receberam dobutamina ${ }^{22}$, corroborando com os achados de Buckberg da relação entre a RVSE e o balanço de oxigênio do território cardíaco. No entanto, nenhum destes trabalhos avaliou pacientes com suspeita de doença arterial coronariana e tentou correlacionar a RVSE obtida pela curva tonométrica com alterações de perfusão cintilográficas.

Um dos motivos pelos quais não foi encontrada diferença no índice em pacientes com ou sem defeito de perfusão coronariana pode estar relacionado com a distribuição dos fatores de risco principais nos dois grupos. A idade, fator de risco cardiovascular importante, apresentou médias acima de 60 anos nos dois grupos de pacientes estudados, tanto aquele com cintilografia normal, quanto naquele com perfusão alterada. Em estudo que avaliou a RVSE entre idosos, hipertensos (com ou sem rigidez aórtica) e grupo controle, que apresentavam média de idade semelhante ao do nosso estudo ${ }^{23}$, o índice de Buckberg também foi semelhante, sendo sugerido que isto se devia a adaptações no intervalo de tempo entre sístole e diástole, que refletem na RVSE, moduladas pela maior pressão aórtica no grupo de idosos hipertensos. Ainda em relação aos fatores de risco do nosso grupo de pacientes, a porcentagem de hipertensos, diabéticos, dislipidêmicos e obesos foi semelhante nos dois grupos, e o impacto desses fatores de risco pode contrabalançar possíveis diferenças relacionadas à perfusão miocárdica avaliada pela cintilografia, principalmente porque esses pacientes, em geral, fazem uso de diferentes tipos de medicações para

\section{REFERÊNCIAS}

1. Chae CU, Pfeffer MA, Glynn RJ, Mitchell GF, Taylor JO, Hennekens $\mathrm{CH}$. Increased pulse pressure and risk of heart failure in the elderly. JAMA 1999;28:634-9. doi:10.1001/ jama.281.7.634. controle dessas comorbidades, as quais podem ter relação direta com o balanço entre oferta e demanda de oxigênio miocárdico. Assim, pode-se constatar que uma limitação do estudo foi a ausência de um grupo controle que incluísse indivíduos jovens com menos fatores de risco. Além disso, no estudo previamente citado que correlacionou a RVSE com isquemia induzível por dobutamina ${ }^{21}$, o índice foi diferente quando comparado durante o pico de infusão do cronotrópico entre os pacientes com ou sem isquemia induzível, entretanto, foi semelhante quando comparado com os valores de RVSE obtidos antes do exame em repouso. Desse modo, isso caracteriza outra limitação do nosso estudo, no qual as medidas de tonometria de aplanação foram obtidas apenas antes do exame cintilográfico, sem avaliação da RVSE durante o estresse farmacológico, no qual poderiam ser detectados valores diferentes de RVSE entre os grupos com cintilografia normal ou alterada.

Apesar de não ser o objetivo principal deste estudo, constatamos que as alterações de perfusão miocárdica foram mais frequentes em homens e nos pacientes com mais de quatro fatores de risco associados. Esse dado tem importância clínica visto que estes pacientes são os de maior risco de doença arterial coronariana grave. Assim, se um paciente tem sintomatologia atípica para doença coronariana e for do sexo masculino e apresentar mais de quatro fatores de risco cardiovascular associados, há alta probabilidade de apresentar defeito de perfusão na cintilografia. No entanto, esse dado deve ser avaliado criticamente, visto que este estudo é transversal e não tem o objetivo de avaliar causa e efeito e, portanto, deve-se confirmar esse achado em estudos prospectivos futuros com grupo populacional maior.

\section{CONCLUSÃO}

Fica evidente pelos nossos dados que a RVSE não pode ser utilizada como parâmetro funcional de perfusão coronariana, visto que não houve diferença entre os grupos estudados. Entretanto, novos trabalhos com obtenção da RVSE durante a cintilografia em estresse, além da presença de grupo controle, devem ser realizados objetivando conclusões mais seguras. Enquanto isso, a RVSE continua refletindo apenas uma diminuição do tempo de diástole para o enchimento das artérias coronárias, que pode ser fator de pior prognóstico para os portadores de hipertrofia miocárdica ou doença coronariana menos grave.
2. Benetos A, Safar M, Rudniche A, Smulyan H, Richard JL, Ducimetiére P. Pulse pressure: a predictor of long-term cardiovascular mortality in a French male population. Hypertension. 1997;30:1410-5. doi: 10.1161/01.HYP.30.6.1410 
3. Darne B, Girerd X, Safar M, Cambien F, Guize L. Pulsatile versus steady component of blood pressure: a cross sectional analysis and a prospective analysis on cardiovascular mortality. Hypertension. 1989;13:392-400. doi: 10.1161/01. HYP.13.4.392

4. Domanski MJ, Davis BR, Pfeffer MA, Kastantin M, Mitchell G. Isolated systolic hypertension: prognostic information provided by pulse pressure. Hypertension. 1999;34:375-80. doi: 10.1161/01.HYP.34.3.375

5. Safar ME, London GM. The arterial system in human hypertension. In: Swales JD, editor. Textbook of hypertension. Oxford: Blackwell; 1994. p.85-102.

6. Cohn JN, Filkenstein S, McVeigh G, Morgan D, LeMay $\mathrm{L}$, Robinson J, et al. Non-invasive pulse wave analysis for the early detection of vascular disease. Hypertension. 1995;26:503-8.

7. Kelly RP, Haywood C, Ganis J, Daley J, Aviola A, O'Rourke M. Non-invasive registration of the arterial pressure waveform using high fidelity applanation tonometry. J Vasc Med Biol. 1989;1(3):142-9. doi:10.1016/0735-1097(92)90198-V

8. Safar M, Peronneau J, Levenson J, Simon A. Pulsed Doppler: diameter, velocity and flow of brachial artery in sustained essential hypertension. Circulation. 1981;63(2):393-400. doi: 10.1161/01.CIR.63.2.393

9. Jondeau G, Botouyrie P, Lacolley P, Laloux B, Dubourg $\mathrm{O}$, Bourdarias JP, Laurent S. Central pulse pressure is a major determinant of ascending aorta dilation in Marfan syndrome. Circulation. 1999;99:2677-81. doi: 10.1161/01. CIR.99.20.2677

10. Boutouyrie P, Bussy C, Lacolley P, Girerd X, Laloux B, Laurent S. Association between local pulse pressure, mean blood pressure, and large artery remodeling. Circulation. 1999;100:1387-93. doi: 10.1161/01.CIR.100.13.1387

11. Nichols W, O'Rourke MF. McDonald's blood flow in arteries. 3rd ed. London: Arnold; 1990.

12. London GM, Blacher J, Pannier B, Guerin AP, Marchais SJ, Safar ME. Arterial wave reflections and survival in end-stage renal failure. Hypertension. 2001;38:434-38. doi: 10.1161/01. HYP.38.3.434

13. Weber T, Auer J, O'Rourke MF et al. Increased arterial wave reflections predict severe cardiovascular events in patients undergoing percutaneous coronary interventions. Eur Heart J. 2005;26:2657-63. doi:10.1093/eurheartj/ehi504

14. Crilly M, Coch C, Bruce M, Clark H, Williams D. Indices of cardiovascular function derived from peripheral pulse wave analysis using radial applanation tonometry: a measurement repeatability study. Vasc Med. 2007;12:189. doi: $10.1177 / 1358863 \mathrm{X} 07081134$

15. Saito M, Kasuya A. Relationship between the subendocardial viability ratio and risk factors for ischemic heart disease. Sangyo Eiseigaku Zasshi. 2003;45(3):114-9. http://dx.doi. org/10.1539/sangyoeisei.45.114

16. Sansoy V, Glover DK, Watson DD, Ruiz M, Smith WH, Simanis JP, et al. Comparison of thallium-201 resting redistribution with technetium-99m-sestamibi uptake and functional response to dobutamine for assessment of myocardial viability. Circulation. 1995;92:994-1004. doi: 10.1161/01.CIR.92.4.99

17. Sociedade Brasileira de Cardiologia, Sociedade Brasileira de Hipertensão, Sociedade Brasileira de Nefrologia. VI Diretrizes Brasileiras de Hipertensão. Arq Bras Cardiol. 2010;95(1 supl.1):1-51. Disponível em: http://publicacoes. cardiol.br/consenso/2010/Diretriz hipertensao associados. pdf

18. American Heart Association Writing Group on Myocardial Segmentation and Registration for Cardiac Imaging. Standardized myocardial segmentation and nomenclature for tomographic imaging of the heart. A statement for healthcare professionals from the Cardiac Imaging Committee of the Council on Clinical Cardiology of the American Heart Association. Circulation. 2002;105(4):539-42. doi: 10.1161/ hc0402.102975

19. Buckberg GD, Fixler DE, Archie JP, Hoffman JI. Experimental subendocardial ischemia in dogs with normal coronary arteries. Circ Res. 1972;30:67-81. doi: 10.1161/01.RES.30.1.67

20. Tsiachris D, Tsioufis C, Syrseloudis D, Roussos D, Tatsis I, et al. Subendocardial viability ratio as an index of impaired coronary flow reserve in hypertensives without significant coronary artery stenosis. J Hum Hypertens. 2012,26(1):64-70. doi: 10.1038/jhh.2010.127.

21. Schultz MG, Abhayaratna WP, Marwick TH, Sharman JE. Myocardial perfusion and the $\mathrm{J}$ curve association between diastolic blood pressure and mortality. Am J Hypertens. 201;26(4):557-66. doi: 10.1093/ajh/hps077.

22. Jellema WT, Groeneveld J, Wesseling KH, Thijs L, Westerhof $\mathrm{N}$, van Lieshout JJ. Heterogeneity and prediction of hemodynamic responses to dobutamine in patients with septic shock. Crit Care Med. 2006;34:2392-8. doi: 10.1097/01. CCM.0000233871.52553.CD

23. Chemla D, Nitenberg A, Teboul JL, Richard C, Monnet X, Clesiau $\mathrm{H}$, et al. Subendocardial viability ratio estimated by arterial tonometry: a critical evaluation in elderly hypertensive patients with increased aortic stiffness. Clin Exp Pharmacol Physiol. 2008;35(8):909-15. doi: 10.1111/j.14401681.2008.04927.x. 